\title{
A Movement-System-Impairment Approach to the Evaluation and Treatment of a Patient with Femoral Anterior Glide Syndrome: A Case Report
}

\author{
Jun-hyeok Jang, Ph.D., PT, Professor ${ }^{1}$; In-cheol Jeon, Ph.D., PT, Professor ${ }^{2}$ \\ ${ }^{1}$ Hill \& Heal Balance Center, Busan, South Korea \\ ${ }^{2}$ Department of Physical Therapy, Hoseo University, Asan, South Korea
}

Background Femoral anterior glide syndrome (FAGS) occurs because of inadequate posterior glide of the femoral head during hip flexion. FAGS can occur with and without medial rotation. The most common form of FAGS occurs with hip medial rotation. The movement-systemimpairment (MSI) approach is based on the association of symptoms with incorrect movements of the hip. The purpose of this case report was to describe the MSI-based approach for the diagnosis of FAGS in a single patient.

Study design Case report.

Observations A 31-year-old female patient had severe pain in the right hip and knee. Four months previously, she complained of pain while walking, which she developed after exercising on the floor for a long time. A clinical test based on the MSI approach led to the diagnosis of FAGS. The patient was instructed to perform an MSI-based special exercise program. Through 5 weeks of the MSI exercise program, changes in muscle length, muscle strength, and movement control of the hip were observed, resulting in reduced pain and improved functional movement.

Conclusions The present study suggests that the therapeutic application of the MSI approach is effective in patients with FAGS.

Key words Femoral anterior glide syndrome; Hip joint; Movement system impairment.
JMST
2018; 2(2): 58-63
Published Online
Dec 31, 2018
pISSN 2635-8573
eISSN 2635-8581

\begin{abstract}
Article History
Received 11 October 2018

Revised 11 October 2018

(1st)

Revised 01 November 2018

(2nd)

Accepted 02 November 2018

\section{CONTACT}

jangpt@hanmail.net

Jun-hyeok Jang ,

Hill \& Heal Balance

Center, Busan,

South Korea
\end{abstract}

\section{This is an Open-Access article distributed under the terms of the Creative Commons Att- ribution Non-Commercial Li- cense (http://creativecommons.
org/licenses/by-nc/4.0) which permits unrestricted non-co- mmercial use, distribution, and reproduction in any me- dium, provided the original work is properly cited.}

\section{INTRODUCTION}

Pain of the anterior hip can have various causes, such as incorrect hip alignment, abnormal movement control, and muscle imbalance. ${ }^{1,2}$ Sahrmann investigated patients with anterior hip pain based on movement system impairment (MSI) and associated their pain with femoral anterior glide syndrome (FAGS). ${ }^{3}$ Patients with FAGS experience anterior hip pain when performing hip flexion, extension, and rotation. Most cases of hip pain are caused by movement impairment, which results in muscle imbalance. ${ }^{2,3}$

During normal hip flexion, a slight posterior glide nor- mally occurs at the femoral head. The posterior glide of the femoral head steadily maintains the path of the instant center of rotation (PICR) of the hip. ${ }^{1}$ However, the posterior glide of the femoral head does not occur appropriately during hip flexion in patients with FAGS, causing inguinal impingement or anterior hip pain.,

The pain and impingement phenomena in hip flexion are caused by movement impairment due to incompatibility of muscle actions. In the hip flexor, imbalance between the iliopsoas and tensor fasciae latae (TFL) muscles occur. Both muscles have the function of hip flexion; however, the TFL is a muscle that performs flexion, abduction, and internal 
rotation of the hip. In patients with FAGS, the muscle action of the TFL is greater than that of the iliopsoas, causing adduction and internal rotation of the hip and resulting in hip pain. ${ }^{3-5}$

The imbalance phenomenon can also be observed in hip abductors. The posterior gluteus medius (PGM) muscle performs extension, abduction, and slight external rotation of the hip. ${ }^{2}$ However, in patients with FAGS, the muscle action of the TFL is greater than that of the PGM, causing internal rotation of the hip when conducting a PGM muscle test. In addition, internal rotation of the hip occurs when standing on one leg. In general, it is believed that internal rotation of the hip may or may not occur in patients with FAGS; however, in most cases, internal rotation of the hip occurs in these patients because the length of their TFL muscle is shorted. ${ }^{3}$

Patients with anterior hip pain experience increased hip pain in the prone position when performing hip extension. To maintain PICR during extension of the hip, the gluteus maximus has to be activated first and to be more dominant than the hamstring. ${ }^{5,6}$ However, if the hamstring action dominates, the femoral head cannot maintain PICR and shifts forward, resulting in anterior hip pain. ${ }^{3,4}$

In case of the hamstring, medial hamstring action and lateral hamstring action have to be distinguished during testing. In patients with FAGS, medial hamstring action dominates. Therefore, when extending the knee while sitting down, the internal rotation of the hip restricts knee extension.

Hence, in patients with FAGS, anterior hip pain is due to improper posterior glide and lack of movement control during hip flexion. ${ }^{3-5}$ To treat FAGS in patients with anterior hip pain, it is important to choose a diagnostic method based on movement impairment and a treatment method that recovers functional movement. ${ }^{7}$

The purpose of this case report is to describe Sahrmann's MSI-based diagnostic approach and the results in a patient with anterior hip pain.

\section{CASE HISTORY}

A 31-year-old female patient experienced pain in the right knee and anterior hip after exercising for 3 hours on a mat for Pilates education 4 months previously. She initially developed anterior knee pain and later developed anterior hip pain. She had a staggering gait due to pain and experienced crepitus of the hip. The knee and hip pain was about level 8 on the visual analogue scale (VAS). ${ }^{8}$ As her discomfort due to hip pain continued when lying down to sleep, and the symptoms of pain while walking worsened and extended to the ankle, she was admitted to the hospital for 3 weeks for treatment. No specific anomalies were found according to the hospital examination, and she was prescribed with analgesics and physical therapy. When the patient visited our center, she had knee and hip pain of VAS 3. She had anterior hip pain when flexing the hip while lying down, and anterior knee pain when flexing and extending while sitting on a table.

\section{PHYSICAL EXAMINATION}

Sahrmann's MSI approach was selected for physical examination. ${ }^{3}$ Examination and treatment were carried out by a physical therapist with 20 years of clinical experience. The examiner examined hip alignment; manual muscle test results; active straight leg raise (ASLR); sitting knee extension; hip joint flexion, adduction, and internal rotation (FADIR); and hip extension in prone position.

The anterior superior iliac spine and posterior superior iliac spine angle of the right hip was measured by the palpation meter while standing and found to be $30^{\circ}$ with $25^{\circ}$ anterior tilt on the opposite side, with internal rotation of the femur (Figure 1). When the examiner connected the iliac crest top and the center of the greater trochanter through palpation, the center of the greater trochanter was located about $2 \mathrm{~cm}$ anterior to the iliac crest top.

The manual muscle testing by Kendall was used to measure the muscle strength of the flexors, abductors, and internal and external rotators of both legs for comparison. According to manual muscle testing, the PGM and external

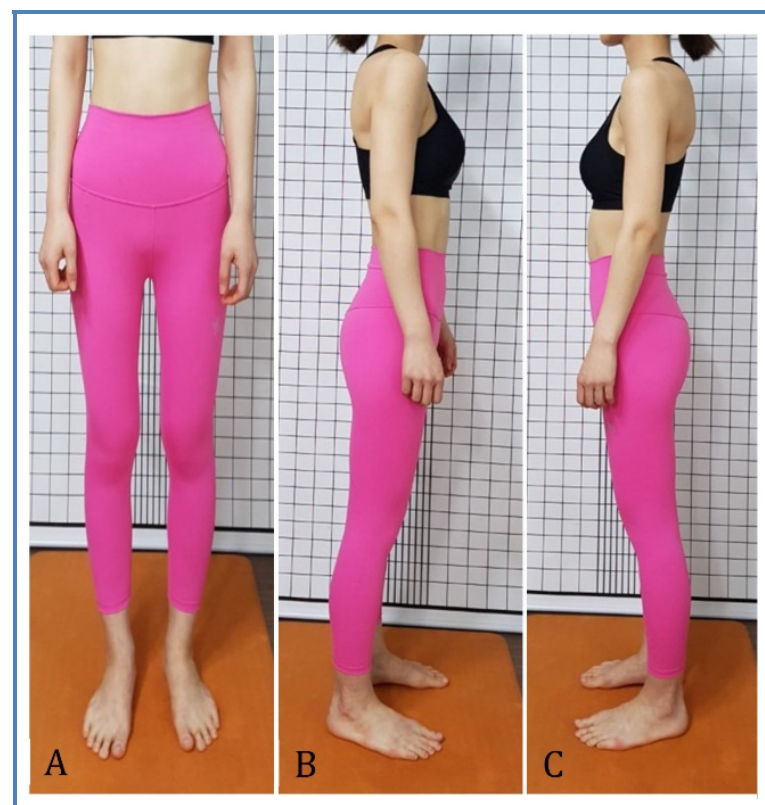

Figure 1. Alignment in standing postures. (A) anterior view, (B) right side view, (C) left side view. 
rotator of the right side were weaker than those of the left side (Table 1).

When performing ASLR while lying down, the hip showed adduction and internal rotation, causing inguinal pain. Even when the patient flexed the hip during ASLR, she experienced inguinal pain.

When the examiner manually performed $90^{\circ}$ flexion, $10^{\circ}$ adduction, and $10^{\circ}$ internal rotation of the hip in a lying position, the patient experienced anterior hip pain (Table 2). This represents a positive FADIR test that shows anterior impingement of the femoral head on the acetabulum. ${ }^{9}$ To prevent the impingement phenomenon, the examiner performed the FADIR test while pushing the greater trochanter for the posterior glide of the femoral head, which reduced the inguinal pain.

When the patient was performing sitting knee extension, the hip showed internal rotation, causing anterior knee pain (Table 2). The pain was reduced when active knee extension was performed while the examiner maintained the neutral position of the hip without internal rotation. In a sitting position, the anterior knee pain disappeared when the patient extended the knee while the examiner pushed down on the greater trochanter.

Performing $10^{\circ}$ active hip extension in a prone position did not cause anterior hip pain. However, the greater trochanter moved forward when the patient extended the hip (Table 2).

\section{DIAGNOSIS}

Considering the results of the above-mentioned tests, the patient's MSI-based diagnosis was FAGS (Table 3). As the results of the patient's examination were consistent with the MSI syndrome, the MSI approach was indicated for the patient's treatment.

\section{TREATMENT}

According to the MSI-based diagnosis, treatment was carried out 10 times over 5 weeks. The patient was in-

Table 1. Manual muscle test: comparison over 5 weeks

\begin{tabular}{ccccc}
\hline Characteristics & Initial right & Initial left & 5 weeks right & 5 weeks left \\
\hline Hip internal rotation & $3+/ 5$ & $3+/ 5$ & $4+/ 5$ & $4+/ 5$ \\
Hip abduction (PGM) & $2+/ 5$ & $3 / 5$ & $4+/ 5$ & $4+/ 5$ \\
Hip flexion & $3+/ 5$ & $3+/ 5$ & $4+/ 5$ & $4+/ 5$ \\
Hip external rotation & $2+/ 5$ & $3 / 5$ & $4+/ 5$ & $4+/ 5$ \\
\hline
\end{tabular}

$5 / 5$, normal strength; $4+/ 5$, able to hold against moderate to strong resistance; $4 / 5$, able to hold against moderate resistance; $4-/ 5$, able to hold against slight to moderate resistance; $3+/ 5$, able to hold against minimal resistance; $3 / 5$, able to hold against gravity but not against additional minimal resistance applied manually; $2+/ 5$, moves through partial range of motion against gravity.

Abbreviation: PGM, posterior gluteus medius.

Table 2. Outcomes of other measures

\begin{tabular}{ccc}
\hline Measure & Initial & 5 weeks \\
\hline VAS & At rest: $3 / 10$ & At rest: $0 / 10$ \\
\hline Pelvic anterior tilt & RT $30^{\circ}$ & RT $20^{\circ}$ \\
LT $25^{\circ}$ & LT $20^{\circ}$ \\
\hline ASLR & RT $40^{\circ}($ pain $)$ & RT $70^{\circ}$ \\
& LT $70^{\circ}$ & LT $70^{\circ}$ \\
FADIR test & Positive & Negative \\
Sitting knee extension & (anterior displacement of the GT) & Negative \\
(RT side) & Positive & Negative \\
\hline Prone $10^{\circ}$ hip extension & (anterior displacement of the GT) & Positive \\
(RT side) & (anterior displacement of the GT) & \\
\hline
\end{tabular}

Abbreviations: VAS, visual analogue scale; ASLR, active straight leg raise; FADIR, hip joint flexion, adduction, and internal rotation; GT, greater trochanter; RT, right; LT, left. 
Table 3. Movement system impairment diagnosis

\begin{tabular}{cccccc}
\hline Diagnosis & Movement impairment & Posture & Muscle strength & Muscle length & Pain \\
\hline & Hip IR during standing & Pelvic anterior tilt & Weak right PGM & Short right TFL & With right hip \\
Femoral anterior & and walking. & Hip extension & Weak hip external & Iliopsoas hamstring & flexion, IR and \\
alide syndrome & Hip adduction and IR & Hip IR & rotators & & \\
& during ASLR & Hion & & & \\
\hline
\end{tabular}

Abbreviations: IR, internal rotation; ASLR, active straight leg raise; PGM, posterior gluteus medius; TFL, tensor fascia lata.

structed to avoid twisting her legs through posture training to prevent weakening of abduction and external rotation of the hip muscles. In addition, she was instructed to place a cushion between the legs when sleeping on the side to prevent excessive adduction and internal rotation.

To correct the muscle imbalance and movement impairment confirmed through the examination, Sahrmann's exercise methods were applied to the patient (Table 4).

The first goal of the 10-session treatment was to increase the length of the shortened TFL, iliopsoas, and hamstring muscles, as well as to continuously strengthen the weakened abductor and external rotator muscles. In particular, we focused on strengthening the posterior part of the abductor. The second goal was to prevent anterior impingement of the hip during functional movement.

\section{OUTCOME}

Reassessment at 5 weeks

After 10 sessions of treatment, the patient gradually showed reduced pain and improved functional movement. The VAS score was 0 out of 10 when resting. The muscles of the lower extremities that were weak before treatment improved. In particular, the muscle strength of the right PGM and hip external rotator increased (Table 1). The anterior hip pain that developed when flexing the right hip while lying down disappeared, and the ASLR angle increased and the pain also disappeared. The anterior hip and anterior knee pain that developed when extending the right hip while sitting disappeared, enabling the patient to comfortably extend the knee. The $10^{\circ}$ of hip extension in a prone position did not cause shifting of the greater trochanter (Table 2). The patient visited our center for treatment 10 times over 5 weeks, with $60 \mathrm{~min}$ of exercise for each visit.

\section{DISCUSSION}

This study examined the method of diagnosing FAGS in a patient with anterior hip pain and the results after 5 weeks of exercise. The results confirmed that the exercises for patients with FAGS suggested by Sahrmann are effective in controlling hip movement and pain.

In patients with FAGS, posterior glide of the femoral head does not appropriately occur when flexing the hip.

Table 4. Intervention: exercises

\begin{tabular}{|c|c|c|c|c|}
\hline Exercise & Instruction & Purpose & Frequency & Repetitions \\
\hline $\begin{array}{l}\text { Quadruped } \\
\text { rock back }\end{array}$ & $\begin{array}{c}\text { On hands and knees, } \\
\text { pull in the abs and rock back } \\
\text { toward heels }\end{array}$ & $\begin{array}{l}\text { To improve hip } \\
\text { flexion and femoral } \\
\text { head posterior glide }\end{array}$ & 2 times per week & $\begin{array}{l}5 \mathrm{~min} \\
\text { Hold for } 5 \mathrm{~s} \text {, rest for } 5 \mathrm{~s}\end{array}$ \\
\hline $\begin{array}{l}\text { Side-lying hip } \\
\text { abduction and ER }\end{array}$ & $\begin{array}{l}\text { Pull in the abs. } \\
\text { Move in the hip joint only } \\
\text { Do not let the pelvis rotate } \\
\text { Alternately for both sides }\end{array}$ & $\begin{array}{l}\text { To strengthen the hip } \\
\text { abductor and external } \\
\text { rotators }\end{array}$ & 2 times per week & $\begin{array}{l}10 \mathrm{~min} \\
\text { Hold for } 10 \mathrm{~s} \text {, rest for } 5 \mathrm{~s}\end{array}$ \\
\hline $\begin{array}{l}\text { Prone hip } \\
\text { extension }\end{array}$ & $\begin{array}{l}\text { Pull in the abs. } \\
\text { Do not allow GT anterior displacement } \\
\text { Alternately for both sides }\end{array}$ & $\begin{array}{l}\text { To strengthen the } \\
\text { gluteus maximus }\end{array}$ & 2 times per week & $\begin{array}{l}10 \mathrm{~min} \\
\text { Hold for } 10 \mathrm{~s} \text {, rest for } 5 \mathrm{~s}\end{array}$ \\
\hline $\begin{array}{l}\text { Sitting knee } \\
\text { extension }\end{array}$ & $\begin{array}{l}\text { Pull in the abs. } \\
\text { Move in the knee joint only } \\
\text { Do not allow the pelvis to } \\
\text { posteriorly rotate } \\
\text { Alternately for both sides }\end{array}$ & $\begin{array}{l}\text { To elongate the } \\
\text { hamstring }\end{array}$ & 2 times per week & $\begin{array}{l}10 \mathrm{~min} \\
\text { Hold for } 10 \mathrm{~s} \text {, rest for } 5 \mathrm{~s}\end{array}$ \\
\hline
\end{tabular}

Abbreviations: ER, external rotation; abs, abdominals; GT, great trochanter. 
Therefore, as the femoral head moves forward, the stretching or impingement of soft tissues in front of the hip causes pain. Sahrmann recommended backward rocking exercise in the quadruped position to cause normal posterior glide of the femoral head. ${ }^{3}$ However, this exercise should be chosen carefully as it elongates the piriformis muscle. If the patient's piriformis is shortened, then it can be an effective method to promote posterior glide of the femoral head and extending the piriformis. ${ }^{10-13}$

In the patient in the present case, the external rotators including the piriformis were very weak. Thus, backward rocking exercise in quadruped position was performed for a short period compared to other exercises, and abductorstrengthening exercise was subsequently conducted (Table 4).

Before treatment, the patient's femur was internally rotated when in a standing position. To identify the cause of internal rotation of the femur, the Ober test was performed, which revealed shortening of the TFL muscle. Therefore, exercises to elongate the TFL muscle and strengthen the PGM muscle were performed. After 5 weeks, the internal rotation of the femur in the standing position was improved and the knee position was normal. Elongation exercise for the TFL muscle and muscle-strengthening exercise for the PGM were effective in improving internal rotation of the femur.

The patient's hip showed the state of increased pelvic anterior tilt in standing position. The iliopsoas acts as a hip flexor. However, if this muscle shortens, hip extension becomes insufficient, which is compensated for by increasing the pelvic anterior tilt and lumbar lordosis. The shortened iliopsoas was confirmed using the Thomas test. However, in patients with FAGS, the anterior displacement of the femoral head could be mistaken for normal or elongated muscle if the femoral head is not accurately fixed.

Hence, in this study, the front of the femoral head was fixed to prevent anterior displacement when examining the length of the iliopsoas. As a result, the patient's iliopsoas was shortened, and iliopsoas elongation exercise was carried out.

In many studies in the literature, relaxation techniques for elongating the shortened iliopsoas resulted in reduced pelvic anterior tilting and lumbar lordosis, as well as increased the extension angle of the transversus abdominis muscle and hip joint. ${ }^{14-16}$ The results of this study also confirmed reduced pelvic anterior tilt after performing iliopsoas elongation exercise (Table 2).

The hip extension exercise in a prone position is recommended for increasing the hip extension angle and muscle activity of the gluteus maximus. However, when a patient with FAGS performs hip extension in a prone position, the muscle activity of the hamstring increases more than that of the gluteus maximus. In addition, the muscle contraction timing of the hamstring is relatively faster than that of the gluteus maximus. Further, when a patient with FAGS extends the hip in a prone position, anterior displacement of the greater trochanter is observed. To increase the muscle activity of the gluteus maximus, many researchers recommend exercises that extend the hip in a prone position. However, in patients with FAGS like in this study, it is important to verify anterior displacement of the greater trochanter when performing hip extension exercise in a prone position.

In the case of severe shortening of the hamstring, it is difficult to accurately control the leg raise exercise in a prone position. Moreover, sitting with a slouch in everyday life causes the hamstring to shorten due to pelvic posterior tilt. In this study, the patient had pain in both the anterior knee and the hip when extending the knee in a sitting position, and shortening of the hamstring was confirmed. However, when the examiner pushed the greater trochanter while the patient actively flexed and extended, the knee pain disappeared. This suggests that the hip and knee pains were caused by the anterior displacement of the femoral head when extending the knee joint while sitting and by the shortened hamstring.

Extending the knee in a sitting position is a good exercise to elongate a shortened hamstring. ${ }^{3}$ However, other studies did not evaluate the hip joint but only confirmed the shortening of the hamstring and performed the exercises. In this study, anterior displacement of the femoral head was prevented and hamstring elongation exercise was performed in a patient with FAGS. After 1 week, the hip and knee pains disappeared, and the patient did not experience any pain even when stretching alone for the remaining treatment period.

After 5 weeks of MSI exercise, muscle strength for hip internal rotation, abduction, flexion, and external rotation increased. In particular, the muscle strength of the right hip PGM and external rotation increased (Table 1). It is believed that the increase of these muscles had a stable influence on the hip and knee. When performing ASLR with the right leg, the pain was reduced and range of motion increased. In the FADIR, sitting knee extension, and hip extension tests, the anterior displacement of the greater trochanter disappeared.

Therefore, this study confirmed that MSI-based diagnosis and exercise are effective for good posture alignment, pain control, and movement control of the hip joint in patients with FAGS.

This study has several limitations. First, it is difficult to 
generalize the results, as only 1 patient was included in the study. Second, as there was no follow-up evaluation, the long-term result is unclear. Future studies with a larger sample size are required. Further research and management methods should be conducted in patients with FAGS.

\section{CONCLUSIONS}

This study described the evaluation and treatment methods for patients with FAGS. For the diagnosis of FAGS, it is important to confirm posture alignment, muscle length, muscle strength, and movement impairment. It is also important for patients to recognize that improper posture and movement cause pain. The results of this study suggest that MSI-based exercises are effective for patients with FAGS

\section{Key Points}

Question Is the movement-system-impairment (MSI)-based approach effective for a patient with femoral anterior glide syndrome (FAGS)?

Findings Application of MSI techniques improved pain and movement in a patient with FAGS.

Meaning We suggest that the MSI-based approach can be used in clinical settings for patients with FAGS.

\section{Article information}

Conflict of Interest Disclosures: None.

Funding/Support: None.

Acknowledgment: None.

\section{REFERENCES}

1. Neumann DA. Kinesilolgy of the musculoskeletal system: foundation for rehabilitation (Third Edition): Missouri: Mosby; 2017.

2. Kendall FP, McCreary EK, Provance PG, Rodgers M, Romani WA. Muscles: testing and function, with posture and pain (Kendall, muscles). Philadelphia: Lippincott Williams \& Wilkins; 2005.

3. Sahrmann S. Diagnosis and treatment of movement impairment syndromes. Elsevier Health Sciences; 2002.

4. Lee D, Vleeming A. An integrated therapeutic approach to the treatment of pelvic girdle pain. Movement, Stability \& Lumbopelvic Pain (Second Edition): Elsevier; 2007:621-638.

5. Comerford M, Mottram S. Kinetic control: the management of uncontrolled movement. Elsevier; 2012:415-503.

6. Janda V. Evaluation of muscular imbalance. In: Liebenson $\mathrm{C}$, editor. Rehabilittion of the spin: a practitioner's manual. 1st ed Baltimore: Lippincott Williams \& Wilkins; 1996:97-112.

7. Harris-Hayes M, Van Dillen LR, Sahrmann SA. Classification, treatment and outcomes of a patient with lumbar extension syndrome. Physiotherapy Theory and Practice. 2005;21(3):181-196.

8. Price DD, Bush FM, Long S, Harkins SW. A comparison of pain measurement characteristics of mechanical visual analogue and simple numerical rating scales. Pain. 1994;56(2):217-226.

9. Kuhlman GS, Domb BG. Hip impingement: identifying and treating a common cause of hip pain. American Family Physician. 2009;80(12):1429-1434.

10. Kirschner JS, Foye PM, Cole JL. Piriformis syndrome, diagnosis and treatment. Muscle \& Nerve. 2009;40(1):1018.

11. Mondal M, Sarkar B, Alam S, et al. Prevalence of piriformis tightness in healthy sedentary individuals: a cross-sectional study. IJHSR. 2017;7(7):134-142.

12. Kirschner JS, Foye PM, Cole JL. Piriformis syndrome, diagnosis and treatment. Muscle \& Nerve. 2009;40(1): 10-18.

13. Mondal M, Sarkar B, Alam S, et al. Prevalence of piriformis tightness in healthy sedentary individuals: a cross-sectional study. IJHSR. 2017;7(7):134-142.

14. Malai S, Pichaiyongwongdee S, Sakulsriprasert P. Immediate effect of hold-relax stretching of ilippsoas muscle on transverse abdominis muscle activation in chronic non-specific low back pain with lumbar hyperlordosis. $J$ Med Assoc Thai. 2015;98(Suppl 5):S6-11.

15. Shiwani NR, Keerthi R, Neha SR, et al. Comparision of muscle energy technique and post isometric relaxation on iliopsoas tightness to improve flexibility in healthy young individuals. Int J Appl Res. 2017;3(3):16-21.

16. Chance-Larsen K, Littlewood C, Gart A. Prone hip extension with lower abdominal hollowing improves the relative timing of gluteus maximus activation in relation to biceps femoris. Manula Therapy. 2010;15:61-65. 